\title{
Los talleres básicos de la Universidad de Lima: algunas reflexiones en torno a los procesos de enseñanza-aprendizaje
}

\author{
Martín Fabbri García / Katerine Tapia Vila \\ Universidad de Lima, Perú
}

Recibido: 24 de noviembre de 2013 / Aprobado: 15 de enero de 2014

El presente artículo ha sido elaborado por un grupo de docentes de una de las secciones del curso Proyectos de Arquitectura I y II de la Universidad de Lima. Aborda el tema de la organización del proceso de enseñanza-aprendizaje en los talleres básicos de diseño, proponiendo algunos puntos referenciales que surgen del análisis de los resultados obtenidos en los primeros cuatro años de labor académica. Se proponen las tres fases del proceso del taller, que son: la etapa de motivación o pulsión creativa, el proceso de diseño y la evaluación.

arquitectura, enseñanza-aprendizaje, creatividad, motivación

The basic workshops at the University of Lima: some reflections on the teaching-learning process

This article was prepared by a group of teachers of the courses of Architectural Project I and II from the University of Lima. It aims to address the issue of organizing the teaching-learning process in basic design workshops proposing some reference points from the analysis of the results of the university in these first 4 years of academic work. The 3 phases of the workshop process that are the step of motivation or creative drive, the process of design and evaluation are proposed.

architecture, teaching-learning, creativity, motivation 
La introducción al mundo de la arquitectura supone una serie de variables en donde convergen el arte y las ciencias y que dan como resultado una creación física producto de un procedimiento mental. Para insertar al estudiante de arquitectura en este mundo se deben contemplar muchos aspectos que van desde lo empírico a lo reflexivo, de lo técnico a lo conceptual, de lo real a lo abstracto. Dentro de estos aspectos la creatividad juega un rol primario, que permite resolver problemas de diseño empleando tanto actividades cognitivas como procesos creativos. Por lo tanto, es necesario en esta fase de introducción emplear mecanismos de motivación que permitan al alumno desarrollar su creatividad, entendiendo que esta última es el motor fundamental para la formación de los arquitectos en los talleres básicos. Les toca a los docentes ejercer un rol importante como acompañantes y guías de esta exploración.

En la búsqueda de un adecuado proceso de aprendizaje en la arquitectura, encontramos que el desarrollo de la habilidad creativa es considerado un aspecto esencial en la formación de los jóvenes arquitectos. La dinámica de este proceso tiene como antecedente histórico la visión hegemónica de la École des Beaux Arts de París que centraba la atención de la formación básica en el curso de taller de diseño. En él se transferían directamente los conocimientos y la experiencia de los docentes a los estudiantes por medio de la crítica personalizada de los trabajos de los alumnos: estos escuchan, emulan y trabajan en sus diseños basándose en el método del "aprender haciendo" (Schön, 1983) ${ }^{1}$. La experiencia del docente, en este

1 Donald Schön (1930-1997). Graduado de la Universidad de Harvard con una tesis doctoral sobre John Dewey, modelo, se impone como condición sobre las consideraciones personales del alumno. En los modelos más recientes se considera al estudiante de arquitectura no solo como receptor de información, sino como el actor principal de este proceso valorando sus experiencias y su propia forma de ver el mundo. Investigadores del campo de la creatividad y cognición sugieren que la resolución de problemas de modo innovador requiere del uso de procesos cognitivos que puedan jugar un rol crítico en la producción de ideas excepcionales, es decir, la participación del estudiante de arquitectura no está basada únicamente en la experiencia del docente, sino, en un alto porcentaje, en la experiencia propia.

Las investigadoras Ligia Arévalo, Magaly Bustos, Diana Castañeda y Nicolás Montañez resumen los lineamientos del libro Creative cognition (Finke, Ward y Smith, 1992) y definen los cinco principios que buscan explicar la creatividad desde un punto de vista cognitivo-creativo:

- La creatividad no es un proceso único sino el resultado de muchos tipos de procesos mentales, cada uno de los cuales ayuda a asentar las bases de insight o iluminación creativa.

- Los procesos dan lugar a las estructuras, pero son diferentes a estas.

del cual retomó la idea rectora de "aprender haciendo" y en base a dicho axioma construyó su propuesta. Su aporte a la educación lo podemos catalogar dentro de los supuestos del "aprendizaje reflexivo" o "educación experiencial". La propuesta de Schön asevera que el joven que acude a una universidad logrará las competencias pertinentes más en la práctica (atendiendo pacientes en el caso de estudiantes de psicología 0 acudiendo a las aulas con alumnos reales en el caso de futuros docentes) que lo que se pueda enseñar en las clases teóricas. 
- El enfoque de cognicióncreativa busca identificar las propiedades de las estructuras preinventivas que emergen de la búsqueda creativa y la exploración.

- La diferencia es que la cognición creativa da lugar a una idea de la cualidad o valor de la idea en sí misma.

- Los autores prefieren identificar las condiciones bajo las cuales ocurre el descubrimiento creativo, más que tratar de predecir la actuación creativa de manera absoluta o determinada. Para mejorar la comprensión de estos principios, se hace una conceptualización de este modelo cognitivo.

Este creciente interés por la motivación y el desarrollo de la creatividad en los estudiantes de diseño arquitectónico es analizado también por Hernán Casakin y Shulamith Kreitler en su ponencia del VI Encuentro Latinoamericano de Diseño realizado en el año 2011². En este planteamiento se rescata la idea de considerar el taller de arquitectura como un ámbito educativo y social, donde los estudiantes quedan expuestos a una multiplicidad de opiniones,

2 La ponencia está incluida en el volumen que reúne las comunicaciones enviadas especialmente para el encuentro sobre diseño en la Universidad de Palermo (Argentina). El corpus de dicho volumen está integrado por contribuciones que describen la experimentación, la innovación y la creación y analizan estrategias, estados del arte específicos, modelos de aplicación y aportes científicos relevantes sobre la disciplina del diseño en el contexto argentino y latinoamericano. Desde múltiples perspectivas diagnósticas e interpretativas, los aportes enfatizan la reflexión sobre los objetos disciplinares, las representaciones y expectativas del diseño como disciplina en sus diferentes especificidades. A su vez, las producciones reflexionan sobre la vinculación del diseño con la enseñanza y los procesos de creación producción e investigación como experiencia integrada a las dinámicas de la práctica profesional real. ideas, experiencias y creencias por parte de sus docentes y dentro del cual el pensamiento creativo es un componente fundamental para la creación de productos de diseño; así también, dentro de la investigación se resalta el desarrollo de las habilidades creativas para la resolución de problemas de diseño, convirtiéndolo en un objetivo primario en el proceso de aprendizaje, es decir, no se evalúa la creatividad solo en el resultado físico obtenido, sino que se presta atención a los aspectos de motivación que han influido en su desarrollo y evolución.

Para definir las bases de esta dinámica de aprendizaje, los autores aplicaron un cuestionario de orientación cognitiva de creatividad (COQ-CR) (Kreitler y Kreitler, 1990), que fue diseñado para medir la intensidad y la estructura de la motivación para la creatividad. Los participantes fueron 112 estudiantes de una escuela de arquitectura en un centro universitario en el centro-este de Israel (52 de arquitectura y 60 de ingeniería) a quienes les fue administrado el cuestionario de orientación cognitiva de la creatividad.

El objetivo del estudio fue ofrecer un mejor entendimiento de los principales aspectos que caracterizan la motivación para la creatividad. Para ello se determinó cuatro tipos de componentes cognitivos -creencias y agrupaciones de temas- considerados significativos para la evaluación de la motivación para la creatividad: creencia sobre uno mismo, creencia sobre metas, creencia sobre reglas y normas, y creencia sobre la realidad. Estas cuatro creencias, a su vez, se agruparon en dos factores: el factor interior del estudiante, compuesto por las dos primeras creencias y el factor del medio ambiente compuesto por las dos siguientes. De acuerdo con los resultados de la encuesta se observó que el factor más importante en los estudiantes de 
arquitectura fue el relacionado con las creencias sobre las metas y sobre uno mismo, estos dos tipos de creencias estaban compuestas por aspectos de autodesarrollo: acentuación de la individualidad del ser, autoexpresión, autoexigencia, contribuir a la sociedad y énfasis en el mundo interior. El factor débil de la encuesta fue el relacionado con el medio ambiente:libertad en el funcionamiento, ser receptivo al medio ambiente, absorber del medio ambiente, actuar bajo condiciones de incertidumbre, no funcionalidad y direccionalidad hacia el interior. Ante estos resultados la investigación concluye que la motivación para la creatividad de los estudiantes de arquitectura radica en el ser, en su singularidad, desarrollo y expresión personal. Por lo tanto, "mantener una apertura hacia el medio ambiente pero sin poner en peligro la direccionalidad hacia el interior del estudiante" (Casakin \& Kreitler, 2011), es lo que sugieren los autores, afirmando que un problema actual en la dinámica del proceso de aprendizaje es que los esfuerzos de los docentes en los talleres se centran en la transmisión y adquisición de conocimiento práctico-profesional, o en la promoción y desarrollo de las habilidades creativas. En consecuencia, el potencial creativo latente en la mayoría de los estudiantes llega a ser difícilmente identificado.

Frente a esta nueva dinámica en el proceso de aprendizaje y la importancia del desarrollo de las habilidades creativas en este, se ha generado una intensa discusión para evaluar las competencias que deberían desarrollar los talleres de arquitectura, especialmente los impartidos en el primer año de la carrera. Como conclusión de las reuniones de coordinación del Área de Talleres Básicos de la Universidad de Lima en las que los autores hemos participado, podemos definir dos grandes posturas que enuncian los factores predominantes en la enseñanza de arquitectura: la de los docentes que defienden los factores del medio ambiente, el lugar y el contexto como indispensables para la formación inicial del estudiante de arquitectura; la otra postura sustenta que es necesario introducir al estudiante al medio paulatinamente, haciendo mayor hincapié en los conceptos arquitectónicos básicos y en el factor interior del estudiante, enriqueciendo la propuesta con una experiencia reflexiva y personal.

En este ámbito, nuestro interés como docentes de la Carrera de Arquitectura de la Universidad de Lima en el proceso de aprendizaje se basa en la importancia de la exploración paulatina del estudiante en la lógica proyectual, definiendo el rol del docente como guía de este proceso, impartiendo distintas opciones para fomentar una capacidad crítica y de elección del estudiante y resaltar la importancia de la evaluación como mecanismo de autoafirmación del alumno.

En las siguientes líneas expondremos parte de la metodología de trabajo de uno de los talleres básicos de arquitectura de la Universidad de Lima $^{3}$. El planteamiento general que se lleva a cabo en el primer año de la carrera de arquitectura considera tres grandes momentos de desarrollo en los contenidos semestrales. En primer lugar, la situación motivacional y previa al proyecto, en donde se desencadenan las pulsiones creativas $^{4}$

3 El equipo de trabajo de la sección de Taller de Proyectos involucrado en este artículo está compuesto por los arquitectos Martín Fabbri García, Katerine Tapia Vila, Iván Yalán Reyes, Octavio Montestruque Bisso y Ana Rodríguez Ferrari, y conforma una de las cuatro secciones de la Carrera de Arquitectura de la Universidad de Lima.

4 En el capítulo VI, del libro El método III. El conocimiento del conocimiento. Libro Primero: Antropología del conocimiento (2002), Edgard Morín define una pulsión creativa como una energía profunda que orienta el comportamiento hacia un fin y se descarga al conseguirlo. 


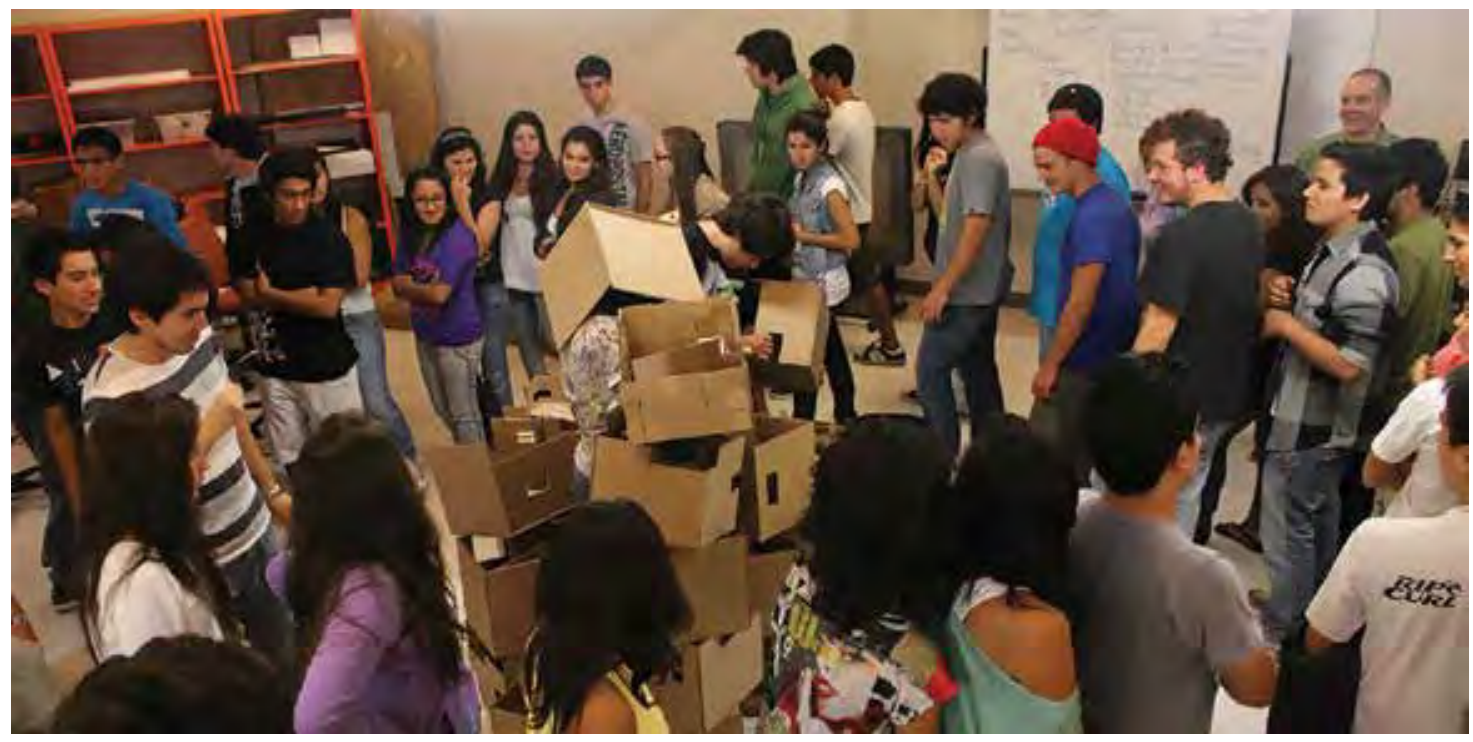

Figura 1.

Esquisse - Niveles I y II. Semestre 2011-1. Exploración del espacio en forma colectiva teniendo como temática central el movimiento y el desplazamiento del cuerpo humano, guiados por el artista Iñaki Peña Zuloaga.

Fotografía: Taller de Modelación.

del estudiante. El segundo momento incluye las estrategias procedimentales para que el estudiante lleve a cabo una experiencia de diseño creativa. $\mathrm{Y}$ en tercer lugar, el momento en el que el taller, como colectivo, evalúa los resultados obtenidos y la forma como fueron procesados.

\section{PULSIÓN CREATIVA}

En el inicio del proceso de diseño el docente presenta al colectivo una serie de ideas fuerza que servirán para impulsar al estudiante a reflexionar sobre su proyecto. En ese momento es fácil pensar que el estudiante visualiza una relación contradictoria entre lo inalcanzable del proyecto, la complejidad de aquello que observa como referente y la posibilidad de autogenerar un desafío que se decide enfrentar. El uso de imágenes de arquitectura está dirigido siempre a lograr en el estudiante una capacidad de analizar las dificultades del proyecto y las posibles rutas por las cuales se puede obtener un resultado creativo, el lenguaje arquitectónico de los referentes no es una condición previa del proyecto.
Aquí se reafirma aquel factor mencionado en la primera parte, por el cual el estudiante obtiene una mayor motivación creativa visualizándose como el sujeto principal del proceso creativo, analizando variables y proyectando la resolución del problema de diseño. Este momento inicial incorporara la variable lúdica para introducir al estudiante en una experiencia de interacción comunicativa; por lo tanto, se establecen reglas, penalidades y retos, que estructuran la base competitiva del "juego". La cátedra asume el liderazgo del grupo acotando las dimensiones de la ruta creativa, visualizando el proceso como una serie de ensayos alternativos y que desembocarán en una serie de resultados finales, sin asumir el proceso como un desarrollo lineal de bases

5 Félix Etxeberria Balerdi, en su artículo "Videojuegos y educación", analiza el valor de los videojuegos como instrumentos de aprendizaje y refiere que lo fundamental de una estructura de aprendizaje basada en lo lúdico es que tenga en sí misma el suficiente atractivo o motivación para promover el aprendizaje. Al igual que en el caso de la arquitectura, se requiere de reglas básicas que puedan ser seguidas por el colectivo en favor de un objetivo común. 
rígidas. La cátedra forma parte de la exploración colectiva del proyecto, fomentando la flexibilidad en el proceso de diseño.

Este liderazgo debe ser consciente de que la exploración es un proceso colectivo y que debe estar guiado por el instinto de adaptabilidad, que es la forma como se introduce en el estudiante la relación entre la arquitectura y su entorno. El profesor se define con el colectivo de estudiantes como un acompañante del proceso de enseñanzaaprendizaje: el alumno como protagonista de un proceso de autoformación y el docente acompañando al estudiante en el desarrollo de sus capacidades.
La crisis habitual del estudiante al inicio del proceso lo impulsa a buscar en la cátedra una imagen-objetivo del proyecto solicitado $\mathrm{y}$ limitar el problema a sus consideraciones de dimensión, materia y espacio: ¿tiene que ser de cartón?, ¿cuánto debe medir?, ¿cuándo es la entrega?, ¿van a pedir algo más?, ¿este es el enunciado final o todavía faltan requisitos?

El estudiante está instintivamente dirigido a acotar y, por lo tanto, a cerrar las posibilidades combinatorias del proyecto y pretende establecer una ruta única que suponga la certeza. La cátedra no busca asegurar un camino único, que lleve al estudiante a obtener un resultado predecible o

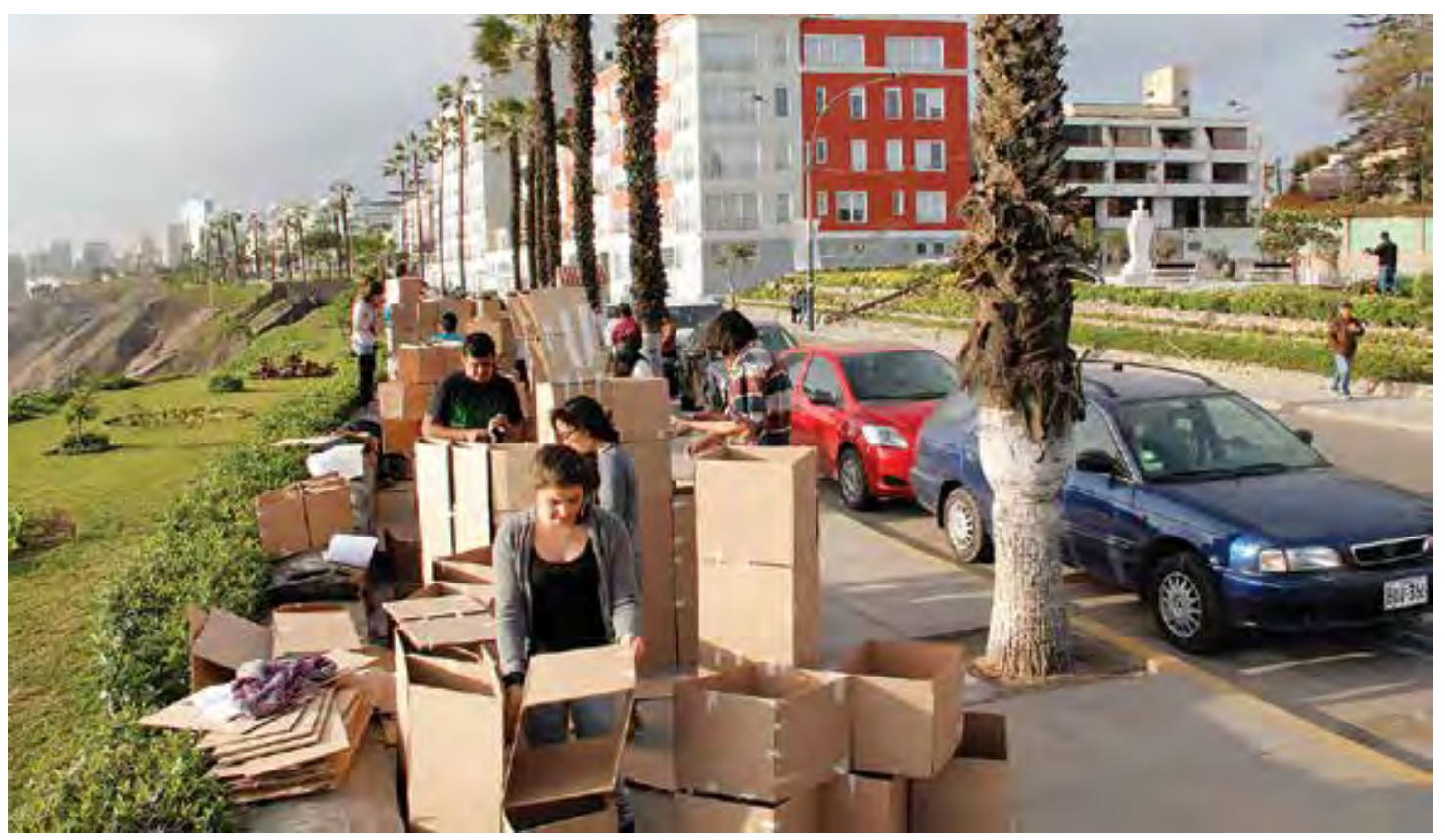

Figura 2.

Esquisse - Niveles I y II. Semestre 2011-2.

Con unos simples cubos de cartón se produce una dinámica en la cual se trabaja la permeabilidad y porosidad de un elemento urbano que se relaciona con la espacialidad del Malecón de Barranco.

Fotografía: Martín Fabbri García. 
reconocible desde el inicio del proceso y que le garantice una eficiencia creativa univalente.

Nosotros requerimos que, desde el inicio, el alumno considere el proceso como un camino por descubrir, y confiamos que estas pulsiones creativas desaten en el estudiante una "ansiedad vital" de conocimiento y, al final de ese proceso cognitivo, una satisfacción en haber recorrido junto con sus compañeros múltiples caminos para obtener el/los resultados finales. Pulsiones, como la base de un proceso intenso en donde la creatividad involucre la concepción de uno como sujeto, pero además de lo "otro" como sujeto alternativo.

\section{EL PROCESO DE DISEÑO}

En este segundo momento es necesario alertar al estudiante acerca de las características del proceso que va a experimentar e impulsarlo a "descubrir" cada etapa del aprendizaje como parte de un proceso autónomo creciente, generando una conciencia crítica y reflexiva.

La autonomía de este momento requiere que el alumno encuentre sus propias respuestas en la experimentación de soluciones. A diferencia del momento inicial, donde se visualizan las estrategias, en esta etapa del proceso es necesario impulsar al estudiante en la elaboración de tácticas de diseño. La forma de descubrir diferentes opciones y validarlas para su proceso sugiere que el alumno convierta las experiencias de sus compañeros en una experiencia propia, evaluando críticamente cada producto como una alternativa más de diseño. Es importante que considere que la solución creativa no es única, sino múltiple. La conciencia crítica en el proceso apunta a que el alumno vea su posición en una constelación de respuestas creativas.
En este sentido, es importante privilegiar la relación del objeto con el "otro", lo alternativo y lo distinto como base de la crítica y plantear los trabajos prácticos como situaciones de acción proyectual, con mecanismos de incertidumbre decreciente, haciendo que el estudiante llegue al producto final por medio de una sucesión de pasos. Con respecto a la definición de lo "otro" podemos citar al profesor Giacomo Rizzolatti en relación con el carácter social del ser humano, confirmado a través del análisis de las "neuronas espejo":

Somos criaturas sociales. Nuestra supervivencia
depende de entender las acciones, intenciones
y emociones de los demás. Las neuronas
espejo nos permiten entender la mente de los
demás, no sólo a través de un razonamiento
conceptual sino mediante la simulación
directa. Sintiendo, no pensando (Artigas,
2009, p. 158). En este proceso la incertidumbre es la actitud por la cual el estudiante concibe cada momento como una etapa de un proceso de cambios y recambios, por lo tanto, cada objeto producido es una unidad, pero a la vez parte de un sistema mayor de unidades que se relacionan con posibilidades infinitas de resultados creativos.

Bajo la teoría de los sistemas de Von Bertalanffy (1976) cada entidad de un orden menor integra a su vez entidades de órdenes superiores. Análogamente, cada trabajo elaborado es una entidad finita y a la vez parte de un proceso mayor de cambio constante.

Consideramos para esta etapa el desarrollo de los contenidos del programa desde núcleos temáticos flexibles que integren: nociones, conceptos y relaciones con experiencias anteriores, contemplando además diversos tipos de clases: debates generales, asesorías personales y discusión en grupos. 


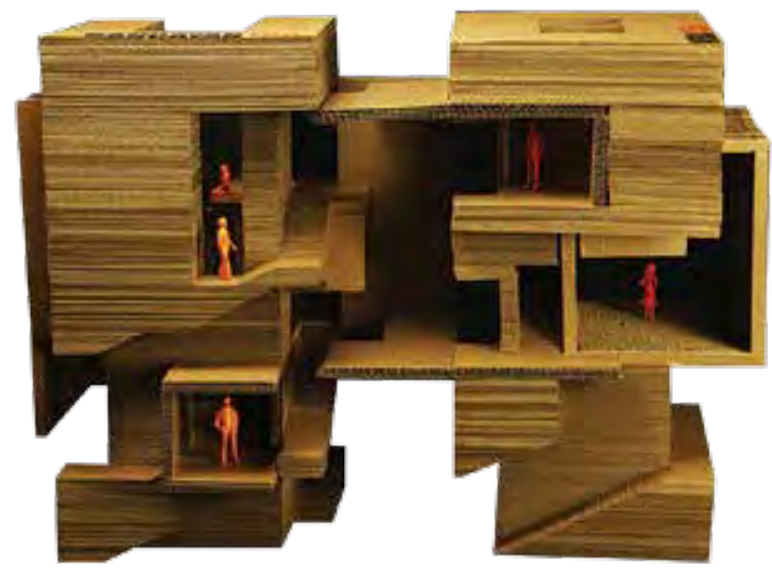

Figura 3.

Torre en movimiento - Nova Vera y Percy Bancayán. Nivel I. Semestre 2013-1.

Entrega final de los proyectos de dos diseñadores que deben producir un objeto único que se ensamble en relaciones y conexiones propuestas por los estudiantes. En este trabajo se experimenta la materialidad y las diversas formas de estructuración, equilibrio y movimiento de la masa.

Fotografía: Taller de Modelación.

Superado el tema motivacional inicial, el proceso requiere sesiones diferenciadas para discutir aspectos específicos de los proyectos:

- Los debates generales, que involucran a toda la colectividad del taller estructurado como una mesa de debate horizontal, donde es importante hablar de la producción propia en relación con la producción colectiva. Bajo este esquema los trabajos son exhibidos como objetos que obligan a los estudiantes a participar comentando los logros obtenidos en el trabajo del compañero.

- Asesorías personales, donde el estudiante requiere entrar en un diálogo con el profesor y recibir de él comentarios, indicaciones, instrucciones o cuestionamientos al trabajo individual. Esta crítica está siempre dirigida a que el alumno descubra posibilidades y aclare sus alternativas de manera autónoma, fomentando el cuestionamiento constante de las bases del proyecto.

- Las discusiones en pequeños grupos de diez alumnos, que permiten brindarle al estudiante un clima de complementariedad entre compañeros, con una participación puntual del docente en la dirección de la sesión y las indicaciones para el trabajo que se debe ejecutar en la clase.

Estas modalidades suponen que la cátedra asuma un intercambio de roles, es decir, que la moderación de los debates, las críticas individuales y las discusiones siempre sean rotativas entre el colectivo docente y el alumno se entrene en un proceso de múltiples opiniones. Lo importante es que él se reafirme como individuo y sepa definir su objeto en relación con otros objetos símiles.

Los núcleos temáticos del curso han sido estructurados considerando que no tienen una jerarquía vertical (uno más importante que el 
otro), o que no tienen una secuencia horizontal (uno después del otro). Los contenidos están planteados como cuatro experiencias anuales diferentes que pueden alternarse indistintamente en tiempo y espacio. El gran objetivo es, pues, aprehender estas cuatro experiencias durante el primer año de la formación de arquitectura. Es allí donde la cátedra juega el rol situacional para ensayar una realidad distinta que envuelva cada experiencia de proyecto. La percepción arquitectónica, la materialidad, la gravedad y el movimiento son las cuatro situaciones en las cuales el proyecto logra obtener de un contexto envolvente ciertas reglas de crecimiento, de evolución, de cambio, de regeneración entendiendo que el contexto no es solamente físico sino también conceptual y cultural-.

Cada una de estas estaciones supone que el proyecto se transforme constantemente, tratando de resolver el cuestionamiento que le propone la gravedad o el desafio que significa el movimiento. Es en esta etapa del proceso donde el alumno considera las condiciones fenoménicas, físicas y matéricas de la arquitectura (peso, fuerza, resistencias, densidades y porosidad). Estos aspectos constituyen la base reflexiva del proceso.

\section{EVALUACIÓN}

El tercer momento de nuestro caso introduce una variable distinta de evaluación que supone un protagonismo mayor del estudiante y del desarrollo de sus capacidades creativas. De esta manera evitamos privilegiar al objeto como producto por evaluar, como acostumbradamente sucede en la formación arquitectónica.

En esta última etapa, la cátedra considera de vital importancia implementar un sistema de evaluación final que suponga la presentación de todo el proceso de diseño realizado durante el ciclo. Esto con la intención de reconsiderar, en un debate abierto, el desempeño de cada uno (cátedra y alumnos) en un proceso complejo de diseño. Con esto queremos buscar que el estudiante sustente las razones de sus proyectos, sostenga las elecciones tomadas a lo largo del semestre y pueda, finalmente, comprender la situación de su proyecto en un marco cultural mayor planteado por la cátedra.

Es la oportunidad para aproximarnos al aprendizaje actitudinal del estudiante, proponiéndole un debate horizontal con los profesores, con la intención de medir sus recursos retóricos, proyectuales y constructivos con los que sustenta sus proyectos.Adicionalmente, pretendemos que se implante en esta etapa un clima multidisciplinario implicando a profesionales de otras especialidades que puedan asesorar al estudiante y ofrecerle un punto de vista alternativo.

La evaluación del producto final por medio de la sustentación puede garantizar una objetividad y un grado de certeza sobre lo aprendido, que permite al estudiante procesar la información y continuar su formación con los estudios posteriores.

Esta etapa requiere la elaboración de un discurso personal por parte del estudiante, que logre articular nociones y conceptos del proyecto $\mathrm{y}, \mathrm{a}$ la vez, que aclare los procedimientos utilizados en su elaboración. Es, además, un requisito fundamental que el estudiante logre conectar su experiencia de diseño con experiencias anteriores y paralelas.Aquí se hace evidente la capacidad del alumno de presentar argumentos que permitan verificar la memoria conectiva que explica su proyecto dentro de un campo cultural mayor de referencias múltiples, dentro del campo de la arquitectura y fuera de este. 

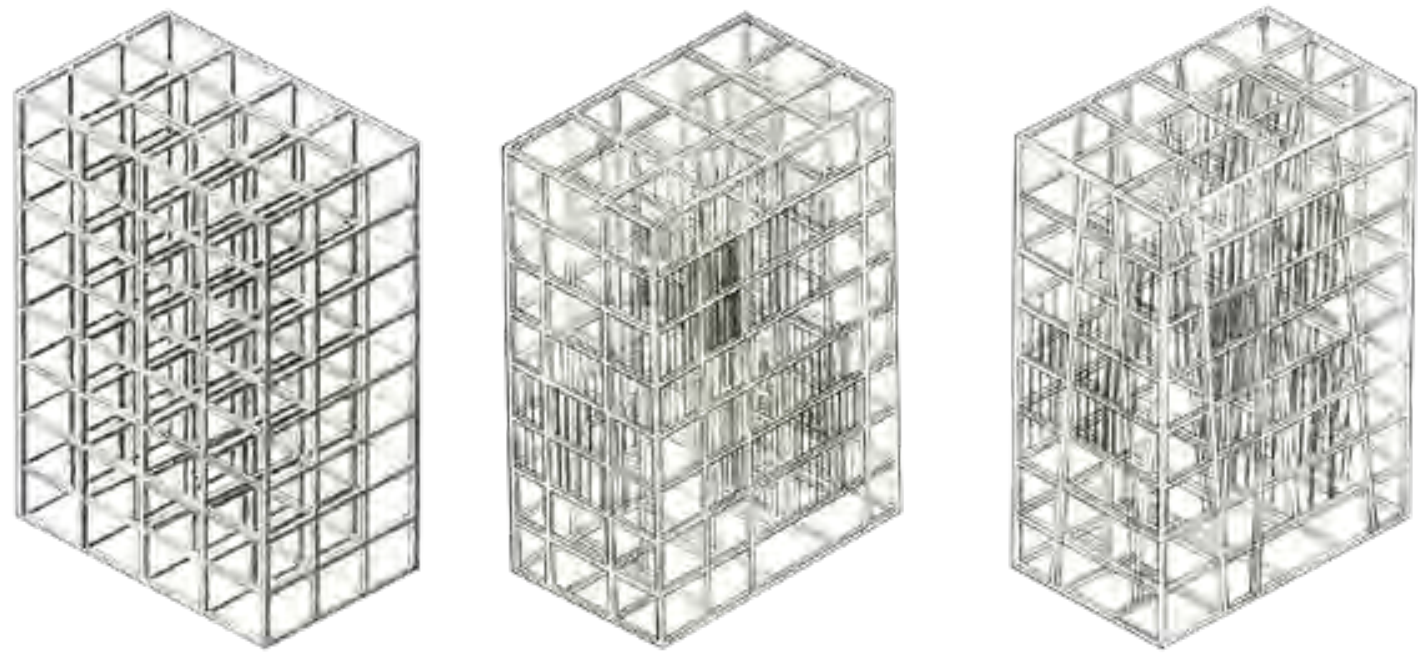

Figura 4.

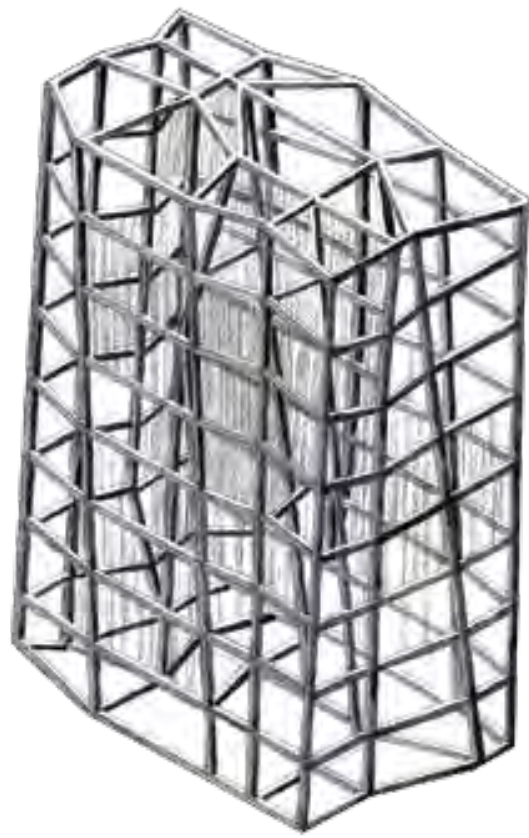

Figura 5

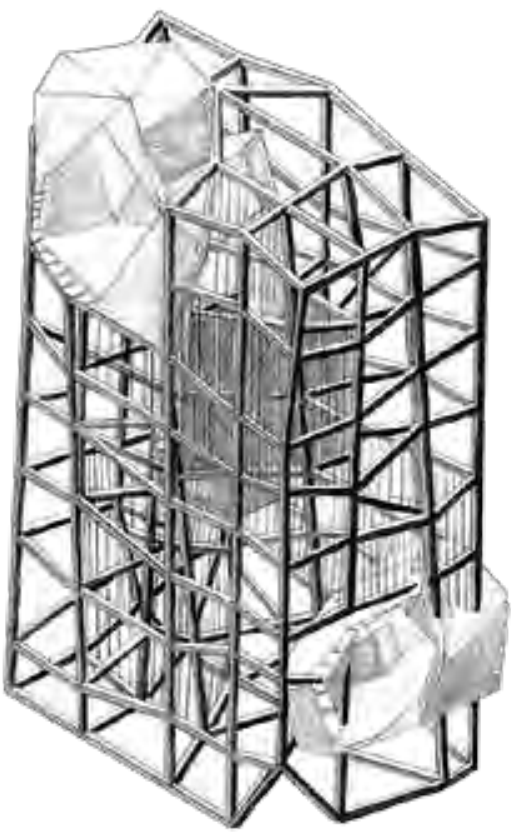




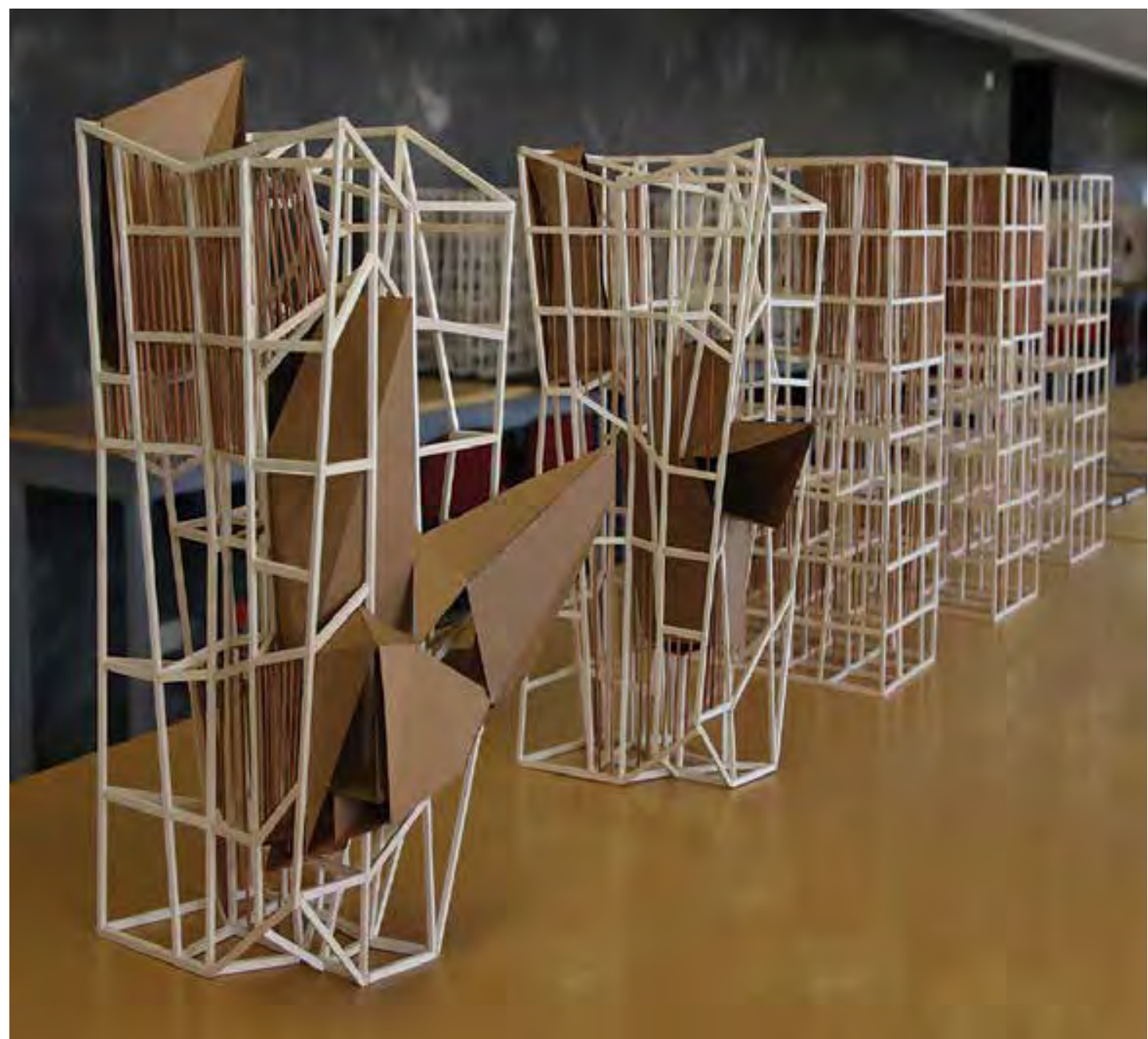

Figura 9

Figuras 4-9.

Torre áurea - Nova Vera. Nivel II. Semestre 2013-2.

Representación isométrica de la estructura inicial propuesta como una estructura uniforme y regular formada por la relación áurea de sus lados. A partir de esta estructura inicial el alumno realiza acciones de desplazamiento controlado de los elementos en una secuencia predeterminada: elementos verticales, elementos horizontales, densificación del tejido que define los planos, rotaciones y liberaciones de elementos estructurales. Cada paso del proceso de diseño es presentado a la clase paulatinamente, creando un ambiente de cambio constante y análisis de variables independientes para garantizar un pleno dominio del proceso por parte del alumno. Como último paso se introducen tres volúmenes con diversas características espaciales y formales (como el uso de la doble capa en la conformación de la envolvente del espacio). El producto final se define en base a un proceso de cinco pasos en donde se explora el movimiento y la gravedad.

Fotografía: Katerine Tapia Vila. 
En la sustentación se procede a la evaluación mediante un esquema de preguntas múltiples que permiten al estudiante abordar su proyecto desde los argumentos-fuerza que él considere necesarios: la plástica del objeto, la pertinencia del concepto en la temática de diseño,la construcción y la materialidad del objeto, etcétera. La cátedra es, en este caso, un receptor de ideas personales, que muestran su coherencia en el momento de la presentación del estudiante y en la necesaria confrontación de ideas.

\section{CONCLUSIONES}

A modo de conclusión, queremos presentar algunas reflexiones finales que nos permiten explicar de mejor manera la experiencia de diseño que hemos tenido en el taller de arquitectura de primer año en la Carrera de Arquitectura de la Universidad

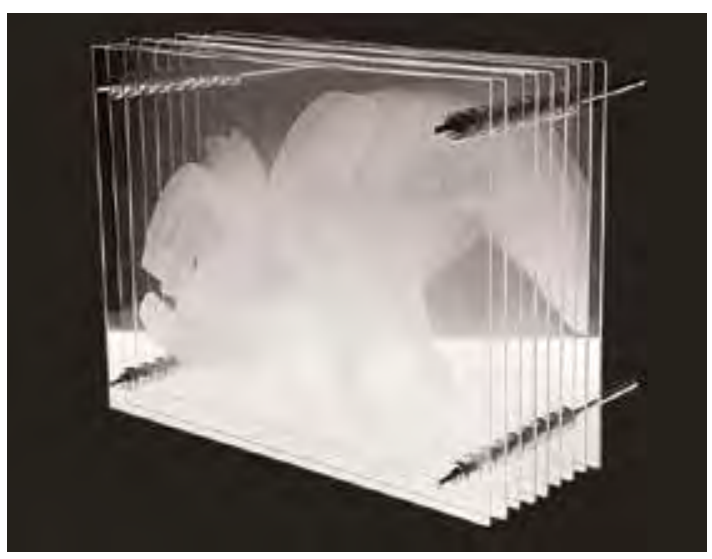

Figura 10.

El capullo - Nivel I. Semestre 2010-2.

Ejercicio de siluetas sucesivas equidistantes que generan un elemento tridimensional a partir de su desplazamiento, dilatación y rotación y que se convierten en un capullo (espacio mínimo) para desarrollar un movimiento humano determinado dentro de él.

Fotografía: Martín Fabbri García. de Lima. Antes de presentarlas como conclusiones cerradas y definitivas, queremos concebirlas como ideas abiertas al debate y a la discusión con las demás secciones que componen la carrera en nuestra universidad:

- Desplegar un proceso de enseñanza/ aprendizaje basado en la dinámica del taller, como una instancia colectiva y social de construcción del conocimiento.

- El proceso tiene como actor principal al alumno, élesquien desarrollalascompetencias en el proceso de autoaprendizaje.

- El rol del docente es acompañar este proceso, introduciendo la motivación durante los momentos claves del desarrollo.

- Es importante reafirmar la motivación como un instrumento de aprendizaje, que busca

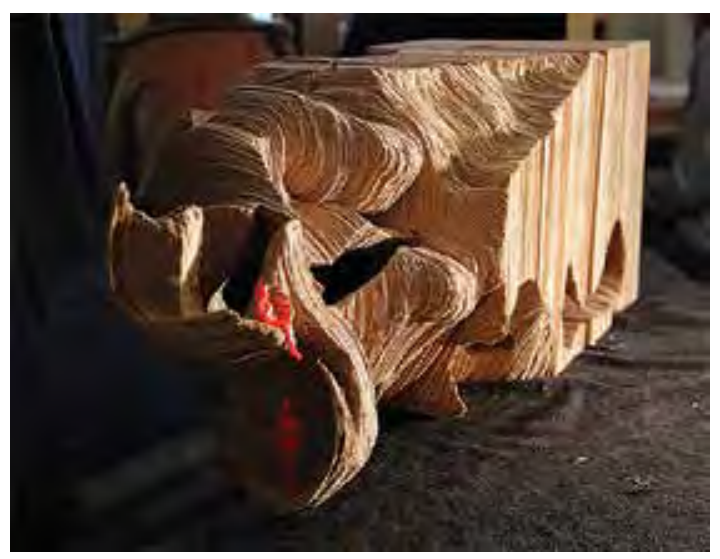

Figura 11.

Elemento macizo-ligero - Alessandra Rodríguez. Nivel II. Semestre 2012-2.

Ejercicio por el cual el alumno explora la transición de lo estereotómico a lo tectónico, partiendo de una masa muy densa que va aligerándose y elevándose del suelo para generar un movimiento orgánico y ambivalente.

Fotografía: Martín Fabbri García. 


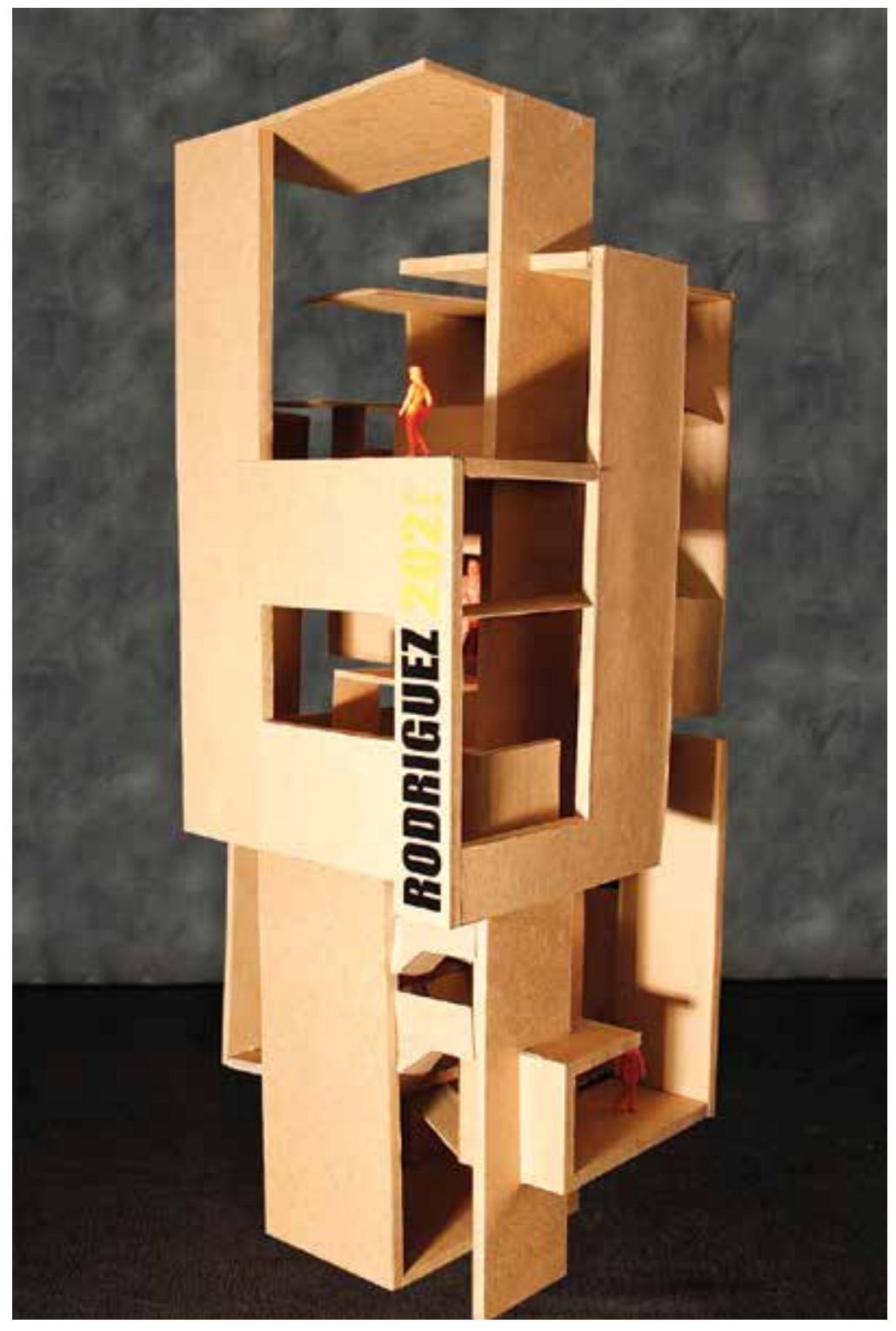

Figura 12.

Torre infinita - Alessandra Rodríguez. Nivel II. Semestre 2012-2.

Exploración espacial, donde se juega con la dinámica del

movimiento a partir de continuidades verticales de apoyos y

elementos de cerramiento espacial.

Fotografía: Martín Fabbri García. 


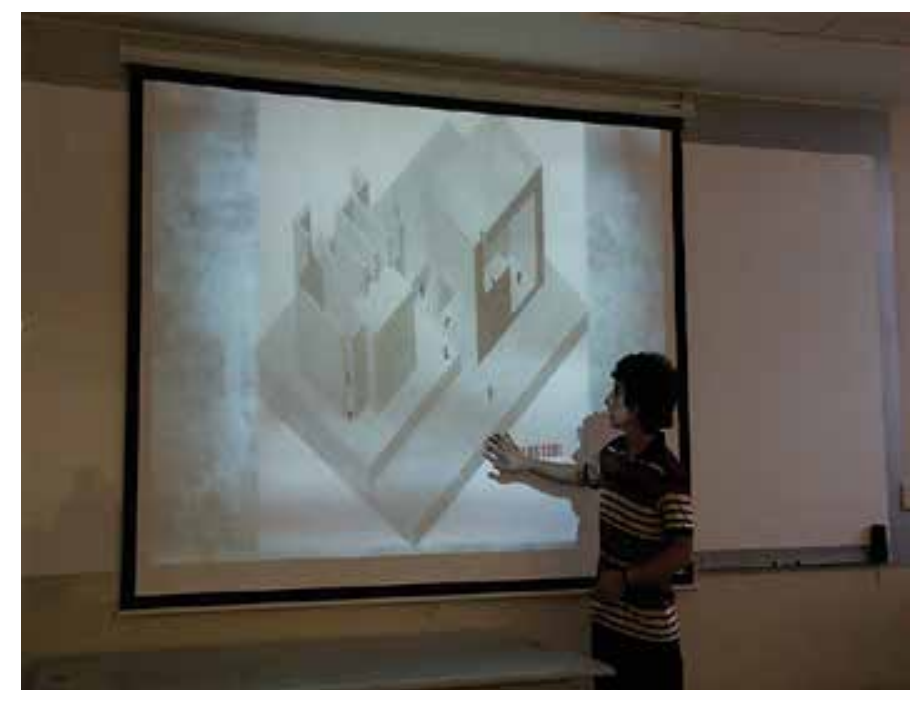

Figura 13.

Sustentación - Raúl Salas. Semestre 2012-1. Hay un énfasis en las sustentaciones por incentivar la reflexión a partir de una gráfica meditada que explore la expresividad de los objetos arquitectónicos.

Fotografía: Martín Fabbri García.

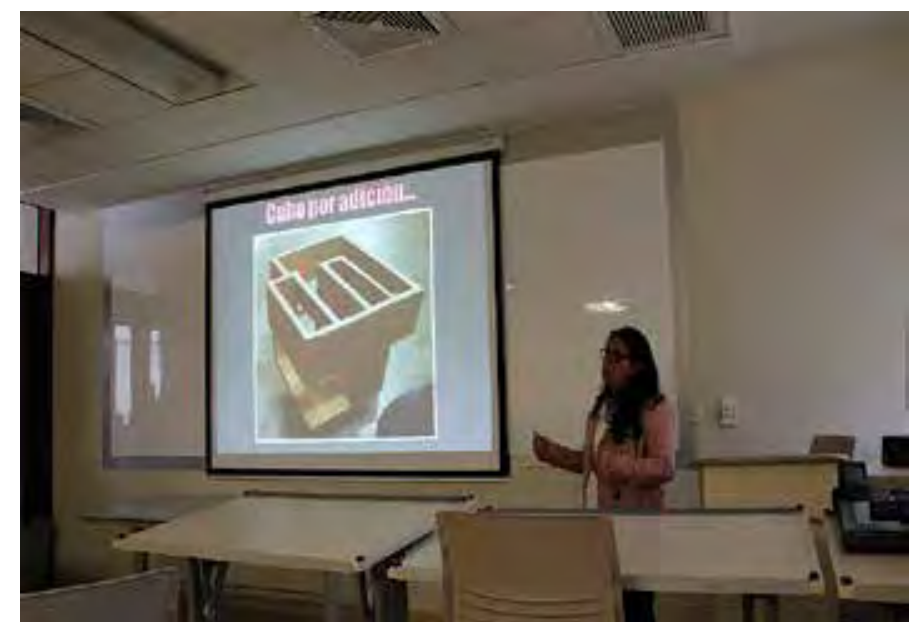

Figura 14

Sustentación - Estrella Caballero. Semestre 2012-1.

Los estudiantes sustentan a partir de su producción las

competencias alcanzadas durante el semestre.

Fotografía: Martín Fabbri García.

Limaq N. ${ }^{\circ} 12015$ ISSN: 2410-6127 
reforzar la individualidad del estudiante y enfatizar los logros alcanzados en su proceso para que estos se conviertan en la base de su conocimiento proyectual.

- El taller sedebe concebir como un laboratorio donde el alumno perciba el error como un requisito del proceso, debe perder el miedo a equivocarse y visualizar la meta mediante aproximaciones sucesivas.

- Debemos sostener que el proceso de diseño tiene como sus características principales la progresividad y la adaptabilidad, entendiendo que la definición final del objeto es parte del proceso y no de un enunciado dado por la cátedra.

- El alumno está obligado a enriquecer el producto propio a través de la concepción de lo "otro" en la arquitectura. Debe concebir la coexistencia de posturas y resultados en el proceso de diseño.

- El proceso está estructurado por momentos de reflexión del estudiante, los cuales se ven reflejados en la sustentación permanente y final del trabajo.

\section{REFERENCIAS}

Arévalo, S. et al. (2009). El desarrollo de los procesos cognitivos creativos a través de la enseñanza problémica en el área de ciencias naturales en niñas del colegio Santa María.Tesis de maestría. Pontificia Universidad Javeriana. Bogotá. Recuperado de http://www.javeriana.edu. co/biblos/tesis/educacion/tesis41.pdf
Artigas Pallares, J.(2009).El niño incomprendido. México, D. F.: Amat Editorial.

Bertalanffy, L. von. (1976). Teoría general de los sistemas: fundamentos, desarrollo, aplicaciones. México, D. F: Fondo de Cultura Económica.

Casakin, H., \& Kreitler, S. (2011). Motivación para la creatividad en estudiantes de diseño: implementación de una estrategia pedagógica. Ponencia presentada en el VI Encuentro Latinoamericano de Diseño. Actas. Vol. 11. Buenos Aires. Recuperado de http://fido. palermo.edu/servicios_dyc/publicacionesdc/ vista/detalle_publicacion.php?id_libro=339

Etxeberria Balerdi, F. (2009).Videojuegos y educación. Teoría de la educación: educación y cultura en la sociedad de la información. Vol. 10 (3). Recuperado de http://gredos. usal.es/jspui/bitstream/10366/56438/1/ TEE2001_V2_videojuevideojuegoseduc.pdf

Finke, R., Ward, T., \& Smith, S. M. (1992). Creative cognition: theory, research, and applications. Cambridge, MA: MIT Press.

Kreitler, K., \& Kreitler, S. (1990). The cognitive orientation and behavior. New York: Springer.

Morín, E. (2002). El método III. El conocimiento del conocimiento. Libro primero:Antropología del conocimiento. Madrid: Cátedra.

Olivera Cárdenas, L.(2012).Docencia universitaria: reflexiones y experiencias. Exposición en el VI Congreso Iberoamericano de Docencia Universitaria. Lima: Pontificia Universidad Católica del Perú, Fondo Editorial. 